\title{
Thermal Flows
}

\author{
Marcello Lappa (D)
}

check for updates

Citation: Lappa, M. Thermal Flows. Fluids 2021, 6, 227. https://doi.org/ 10.3390 / fluids 6060227

Received: 2 June 2021

Accepted: 7 June 2021

Published: 18 June 2021

Publisher's Note: MDPI stays neutral with regard to jurisdictional claims in published maps and institutional affiliations.

Copyright: (C) 2021 by the author. Licensee MDPI, Basel, Switzerland. This article is an open access article distributed under the terms and conditions of the Creative Commons Attribution (CC BY) license (https:// creativecommons.org/licenses/by/ $4.0 /)$.
Department of Mechanical and Aerospace Engineering, University of Strathclyde, James Weir Building, 75 Montrose Street, Glasgow G1 1XJ, UK; marcello.lappa@strath.ac.uk

Flows of thermal origin and heat transfer problems are central in a variety of disciplines and industrial applications. The present Special Issue entitled "Thermal Flows" consists of a collection of studies by distinct investigators and research groups, dealing with different types of flows relevant to both natural and technological contexts. It comprises 11 papers covering some of the latest advances in these fields. Both reviews of the state-of-the-art and new theoretical, numerical and experimental investigations are presented, which illustrate the structure of these flows, their stability behavior and the possible bifurcations to different patterns of symmetry and/or spatiotemporal regimes. Moreover, different categories of fluids are considered (liquid metals, gases, common fluids such as water and silicone oils, organic and inorganic transparent liquids and nanofluids).

The first part of this collection [1-3] focuses on a very classical problem, generally falling under the heading of Rayleigh-Bénard convection (RB), that is, the behavior of fluid systems heated from below and cooled from above. In particular, the first study [1] finds its motivations in the field of thermal comfort in the building heating and ventilation sector, where thermal control is traditionally implemented via floor heating and/or ceiling cooling; interestingly, the response of RB convection to the application of time-periodic cooling is assessed by means of sophisticated LBM simulations. Thermogravitational convection is still at the root of the following two numerical investigations [2,3], where, however, a shift is implemented in the intended outcomes. In particular, the context is changed from the design of heating/cooling systems to the much more fundamental area relating to natural phenomena of a geophysical or astrophysical interest. Indeed, flows of the RB type are also known for their remarkable ability to produce important large-scale features (such as a self-sustained magnetic field) in rotating cosmic objects, relevant exemplars being the sun [2] and terrestrial or gas giant planets [3]. Rotating thermal convection is yet the main subject of analysis in [4], where the non-linear dynamics of such flow are considered on the surface of a hemisphere. It is shown that, just like a half soap bubble located on a heated plate, a hemisphere undergoing rigid or differential rotation can mimic the behavior of large-scale fluid systems (the numerous atmospheric and oceanic flows, which can effectively be modeled as two-dimensional thermal convection on a curved surface).

The next paper on the list [5] is still somehow relevant to typical natural phenomena, as it is concerned with the behavior of a viscous non-isothermal mixing layer of water. In isothermal conditions, such a kind of flow is the simplest configuration allowing for the well-known Kelvin-Helmholtz (or KH) instability that takes place when two parallel currents with different velocities meet. It typically develops in the form of a wave. This study examines the changes that are introduced in these dynamics when the fluid displays a stable thermal stratification.

Paper [6] is a review of the landmark experimental results obtained with regard to a completely different mechanism of convection, which does not require the presence of buoyancy or gravity, namely Marangoni (or thermocapillary) convection. This genus of fluid motion is relevant to a variety of technological sectors (including, but not limited to, soldering processes, film and spray coating and several techniques for the production of crystals of different types). The authors of paper [6] offer a masterful exposition on the state of the art for Marangoni flows in "liquid bridges" (LBs) made of silicone oil and 
other transparent organic liquids (an LB is a column of liquid held by surface tension between two supporting disks at different temperatures). When this type of convection, initially steady and axisymmetric, becomes unstable, it can produce waves travelling in the azimuthal direction that, under certain conditions, can cause the accumulation of tracer particles with various sizes and densities into esthetically appealing structures known as "PAS". Surface tension effects are still central in the next work [7], where the planar laminar free-surface flow of a Newtonian fluid (as typically encountered in slot and blade coating applications) is examined analytically at moderate Reynolds and capillary numbers under adverse or favorable pressure gradients.

Following the treatment of thermogravitational and thermocapillary convection, two studies $[8,9]$ are also included, which concentrate on a third fundamental variant of thermally induced flows, namely fluid motion produced in non-isothermal systems by the application of "vibrations" (thermovibrational convection). Vibrations enable a timevarying acceleration, which formally makes the emerging convective structures a variant of buoyancy convection. As in the parent phenomena, these processes are extremely sensitive to the relative directions of the acceleration and the prevailing temperature gradient. In particular, [8] focuses on a case where these vectors are parallel. The complexity of this specific arrangement (with respect to the companion situation in which vibrations and temperature difference are perpendicular) stems from the properties that are inherited from the corresponding case with steady gravity, i.e., the standard RB. The need to overcome a threshold to induce convection from an initial quiescent state, together with the opposite tendency of acceleration to damp fluid motion when its sign is reversed, causes a variety of possible solutions that can display synchronous, non-synchronous, time-periodic and multi-frequency responses. A very special case is represented by "circular translational vibrations", i.e., a situation where the entire fluid domain moves along a circular path [9]. The peculiarity of this flow originates from the absence of a preferred direction for the vibrations and the existence of a rotating force field in the non-inertial reference frame moving with the fluid container.

The "rotating fluid" theme, already addressed to a certain extent in [9] and in [2-4], returns (although under different perspectives) in [10], where the main purpose is the presentation of an analytical dependence on the critical Reynolds number for a flow near a rotating disk (such a subject being essential for the analysis of the thermal response of disks and blades in relation to the design of gas and steam turbines).

The last work of the sequence [11] finally addresses another important long-standing issue in engineering, that is, the thermal performances of circular pipes with inserts. Such a subject is examined in combination with the use of a nanofluid (water and titanium dioxide) for different values of the Reynolds number. The resulting problem aligns with current efforts aimed to design more efficient heat-exchange technologies to be used in a variety of fields (processor and electronic chip cooling, nuclear reactor cooling, food processing, cooling systems in commercial buildings, just to cite a few).

As witnessed by all of these studies, problems concerned with heat transfer and related (forced or natural) flows continue to burgeon and bring surprises to this day. Along these lines, the focused papers included in this Special Issue have been collected under the optimistic hope that they will serve as a new important resource for physicists, engineers and advanced students interested in the physics of non-isothermal fluid systems, fluid mechanics, environmental phenomena, meteorology, geophysics, thermal, mechanical and materials engineering.

Conflicts of Interest: The author declares no conflict of interest.

\section{References}

1. Nasseri, L.; Himrane, N.; Ameziani, D.E.; Bourada, A.; Bennacer, R. Time-periodic cooling of Rayleigh-Bénard convection. Fluids 2021, 6, 87. [CrossRef]

2. Silva, L.; Gupta, P.; MacTaggart, D.; Simitev, R.D. Effects of shell thickness on cross-helicity generation in convection-driven spherical dynamos. Fluids 2020, 5, 245. [CrossRef] 
3. Simitev, R.D.; Busse, F.H. Onset of inertial magnetoconvection in rotating fluid spheres. Fluids 2021, 6, 41. [CrossRef]

4. Fischer, P.; Bruneau, C.H.; Kellay, H. Numerical study of rotating thermal convection on a hemisphere. Fluids 2020, 5, 185. [CrossRef]

5. Vitoshkin, H.; Gelfgat, A. Non-modal three-dimensional optimal perturbation growth in thermally stratified mixing layers. Fluids 2021, 6, 37. [CrossRef]

6. Ueno, I. Experimental study on coherent structures by particles suspended in half-zone thermocapillary liquid bridges: Review. Fluids 2021, 6, 105. [CrossRef]

7. Khayat, R.E.; Hossain, M.T. Coating flow near channel exit. A theoretical perspective. Fluids 2020, 5, 180. [CrossRef]

8. Crewdson, G.; Lappa, M. The zoo of modes of convection in liquids vibrated along the direction of the temperature gradient. Fluids 2021, 6, 30. [CrossRef]

9. Kozlov, V.; Rysin, K.; Vjatkin, A. Vibroconvective patterns in a layer under translational vibrations of circular polarization. Fluids 2021, 6, 108. [CrossRef]

10. Dmitrenko, A.V. Determination of critical Reynolds number for the flow near a rotating disk on the basis of the theory of stochastic equations and equivalence of measures. Fluids 2021, 6, 5. [CrossRef]

11. Papazian, K.; Al Hajaj, Z.; Saghir, M.Z. Thermal performance of a heated pipe in the presence of a metal foam and twisted tape inserts. Fluids 2020, 5, 195. [CrossRef] 